\title{
Editorial
}

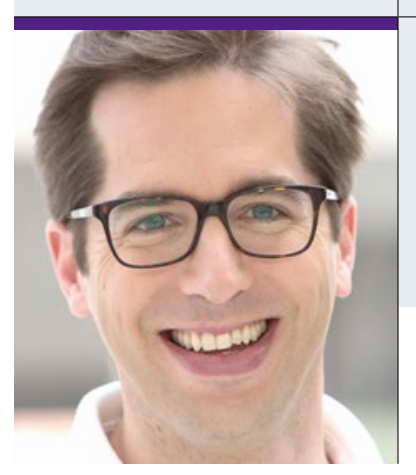

„Es sollte bereits früh nicht nur über die Möglichkeiten aufgeklärt

werden, wie man eine Schwangerschaft verhütet, sondern

ebenso wie lange eine Schwangerschaft überhaupt möglich ist."

\section{Ungewollt schwanger oder kinderlos: ein Aufklärungsappell}

A nhand mehrerer Beiträge werden in dieser Ausgabe sowohl die Notfallkontrazeption als auch Themen aus der Kinderwunschabklärung besprochen. In einem Punkt scheint es, als seien diese Dinge zwei Seiten derselben Medaille: ein Mangel an Information und Aufklärung. Ein Update dieser Themen soll helfen, wichtige Informationen wieder aufzufrischen und auch an unsere Patientinnen weiterzugeben.

Wie im entsprechenden Artikel auf S. 34 ff. beschrieben, wissen offensichtlich nur wenige Schüler über Notfallkontrazeptiva ausreichend Bescheid und die Rate an ungewollten Schwangerschaften sinkt in Ländern mit freier Abgabe nicht. Dies ist umso mehr erschreckend, als durch Entlassung der Notfallkontrazeptiva aus der Verschreibungspflicht nun keine Beratung mehr durch einen Gynäkologen erfolgt und die Zielgruppe diese Medikamente dadurch eventuell im falschen Zeitfenster oder ohne die notwendigen Begleitmaßnahmen wie unmittelbar anschließende Verhütung oder Beginn einer kombinierten oralen Kontrazeption (KOK) einnimmt. Eine Studie aus Schottland, wo nur $43 \%$ der abgebenden Apotheker überhaupt über die Möglichkeit einer anschließenden Verhütung mit KOK berieten, zeigt, dass hier wohl noch immenser Aufklärungsbedarf besteht.

Aber nicht nur in der Notfallkontrazeption besteht ein Mangel an Aufklärung. In einer schwedischen Studie wurde bei einer Befragung von Patientinnen, die über 35 Jahre alt waren und sich einer IVF unterzogen, gezeigt, dass sich immerhin $18 \%$ der Patientinnen des Einflusses des Alters auf die Fertilität nicht bewusst waren. Um hier eine Abschätzung der individuellen Perspektiven der Fertilität zu bekommen, werden vielerorts „Fruchtbarkeitschecks“ angeboten. Dass dieses Thema komplexer ist als es auf den ersten Blick scheint und insbesondere der AntiMüller-Hormon-Wert kein Absolutwert ist, sondern ebenso individuell ausgewertet werden kann, beschreibt ein weiterer Artikel (S. 24 ff.). Aber auch die Themen strukturierte Kinderwunschabklärung, Fertilitätsprotektion bei Tumorerkrankungen und Endometriose sollen in der hier vorliegenden Ausgabe für das Thema Fertilität sensibilisieren und vorhandenes Wissen auffrischen und ergänzen. Denn wenn sich die Patientin beziehungsweise das Paar mit Kinderwunsch zur Abklärung vorstellt, sollte nicht auch noch vonseiten der behandelnden Ärzte durch unstrukturiertes Vorgehen wertvolle Zeit verschwendet werden.

Wenn einerseits die Rate an ungewollten Schwangerschaften trotz freier Abgabe von Notfallkontrazeptiva nicht sinkt und andererseits die Rate an ungewollt kinderlos gebliebenen Frauen steigt, scheint es einiges an Aufklärungsbedarf zu geben. Es sollte bereits früh nicht nur über die Möglichkeiten aufgeklärt werden, wie man eine Schwangerschaft verhütet, sondern ebenso wie lange eine Schwangerschaft überhaupt möglich ist. Denn nur mit dieser Information können unsere Patientinnen eine Familien- und Karriereplanung so angehen, dass der Kinderwunsch nicht irgendwann auf der Strecke bleibt.

\section{Dr. Maximilian Franz}

